\title{
Calculation of Interspike Intervals for Integrate-and-Fire Neurons with Poisson Distribution of Synaptic Inputs
}

\author{
A. N. Burkitt \\ G. M. Clark \\ Bonn Far hustitute, Fast Melboume, Victoria 3002, Australia
}

We present a new technique for calculating the interspike intervals of integrate-and-fire neurons. There are two new components to this technique. First, the probability density of the summed potential is calculated by integrating over the distribution of arrival times of the afferent postsynaptic potentials (PSPS), rather than using conventional stochastic differential equation techniques. A general formulation of this technique is given in terms of the probability distribution of the inputs and the time course of the postsynaptic response. The expressions are evaluated in the gaussian approximation, which gives results that become more accurate for large numbers of small-amplitude PSPs. Second, the probability density of output spikes, which are generated when the potential reaches threshold, is given in terms of an integral involving a conditional probability density. This expression is a generalization of the renewal equation, but it holds for both leaky neurons and situations in which there is no time-translational invariance. The conditional probability density of the potential is calculated using the same technique of integrating over the distribution of arrival times of the afferent PSPs. For inputs with a Poisson distribution, the known analytic solutions for both the perfect integrator model and the Stein model (which incorporates membrane potential leakage) in the diffusion limit are obtained. The interspike interval distribution may also be calculated numerically for models that incorporate both membrane potential leakage and a finite rise time of the postsynaptic response. Plots of the relationship between input and output firing rates, as well as the coefficient of variation, are given, and inputs with varying rates and amplitudes, including inhibitory inputs, are analyzed. The results indicate that neurons functioning near their critical threshold, where the inputs are just sufficient to cause firing, display a large variability in their spike timings.

1 Introduction

The stream of action potentials (spikes) generated by a neuron, which is the means by which neurons communicate, plays an important role in neural information processing. The generation of an action potential can be described 
in terms of the membrane potential of the neuron reaching a threshoid vaiue, as in the integrate-and-fire model. This model, which has both a long history (Lapicque, 1907) and wide application (Tuckwell, 1988a), has recently been compared with the Hodgkin-Huxley model for the case where both modeis receive a stochastic input current, and it was found that the threshold model provides a good approximation (Kistler, Gerstner, \& van I femmen, 1997).

One of the most universal characteristics of neuronal behavior is the apparent stochastic nature of the timing of action potentials (Tuckwell, 1988b, 1989). The traditional view has long been that the mean rate of firing of a netron provides an adequate description of the information it conveys (Adrian, 1928). There are, however, a number of instances within the nervous system where the temporal information contained in the timing of individual spikes plays an important role, such as the encoding of sound frequency information in the auditory pathway, where spikes become locked to the phase of the incoming sound wave (see Theunissen \& Miller, 1995, and Carr \& Friedman, 1999, for reviews of temporal coding). There has also been considerable interest in studies of cross-correlational activity of neurons in the risual cortex, which provide data suggesting a functional role for the synchronization of neural activity, as reviewed by (Engel, König, Kreiter, Schilien, \& Singer, 1992; Singer, 1993). This has led to a recent reappraisal of the information that can be contained in the timing of neuronal spikes (Bialek \& Rieke, 1992), and a variety of mathematical models of netwrorks of spiking neurons have been proposed (Abeles, 1982a; Judd \& Aihara, 1993; Gerstner, 1995) in which the timing of individual spikes encodes information (Hopfield, 1995; Gabbian: \& Koch, 1996; Maass, 1996a, 1996b). Studies suggest that the integrate-and-firc mechanism is unable to account for the irregularity observed in the interspike intervals (ISIs) in spike trains from neurons in the visual cortex of behaving monkeys (Softky \& Koch, 1992, 1993). One possible explanation is that the neurons act as coincidence detectors (Abeles, 1982b), which may be made possible with active dendritic conductances (Softky, 1994). However, subsequent studies (Shadien \& Newsome, 1994, 1998) suggest that the observed irregularity may be accounted for by the effect of in hibitory postsynaptic potentials (IPSPs) and that the timing of neuronal spikes conveys little, if any, information.

The resolution of such issues requires the development and analysis of neuronal models that are sufficiently complex to capture the essential features of the neuronal processing, including stochastic input, while still being mathematically tractable. The earliest solution of the integrate-and-fire model that incorporated stochastic activity was to model the incoming postsynaptic potentials (PSPs) as a random walk (Gerstein \& Mandelbrot, 1964). Subsequent developments have largely built on this diffusion approach using stochastic differential equations and the Ornstein-Uhlenbeck Process (OUP). Stein formulated the integrate-and-fire model with stochastic input to include the decay of the membrane potential (Stein, 1965), and a number of other authors subsequently have investigated the model using both 
stochastic differential equations and numerical techniques (luckwell, 1977; Wilbur \& Rinzel, 1982; Lánsky, 1984). These techniques have been used to examine the role of inhibition in the Stein model (Tuckwell, 1978, 1979; Cope \& Tuckwell, 1979; Tuckwell \& Cope, 1980) and a number of other features, such as the effect of reversal potentials (Tuckwell, 1979; Hanson \& Tuckwell, 1983; Musila \& Lánský, 1994).

We present here an alternative formulation of the stochastic integrateand-fire model that allows the analysis of arbitrary synaptic response functions (which describe the time course of the incoming PSP, including a finite rise time and subsequent decay). The first step is the calculation of the probability density of the summed potential, which is given by integrating over the arrival times of the incoming PSP's. The probability density function at any time is calculated in the gaussian approximation (i.e., expanding in powers of the PSP amplitude, $a$, and neglecting terms of order higher than quadratic in the expansion), and it is found to be a gaussian distribution, with the (time-dependent) mean and wiath of the distribution, depending on the details of the distribution of PSP arrival times. In the case of a Poisson distribution of PSP arrival times, our results reproduce the known expression for the probability density of the membrane potential in the diffusion limit. In order to calculate the probability density of output spikes. it is necessary to know when the potential reaches threshold for the first time This first-passage density is usually related to the probability density of the potential by a renewal equation (luckwell, 1988b), a procedure that works only when there is no decay of the membrane potential (the perfect integrator model). However, it is possible to formulate a more general integral relationship involving the probabilitv density of output spikes and a conditional probability density of the potential (Plesser \& Tanaka, 1997; Burkitt \& Clark, 1999a). We show how this conditional probability density can aiso be calculated using the technique of integrating over the distribution of arrival times of the afferent PSPs. In addition to reproducing the results of the perfect integrator and Stein models, this method allows us to investigate models that incorporate not only the membrane potential leakage but also a finite rise time of the postsynaptic response. Numerical solutions of the spike output distribution (i.e., the interspike interval distribution) of these models are given.

We have also applied this technique to the study of the synchronization problem, in which the relationship among a number of approximately synchronized inputs (with a spread of arrival times giving the temporal fitter of the inputs) with the spread in time of the output spike distribution is examined (Burkitt \& Clark, 1999a). The analysis supports previous studies (Bernander, Koch, \& Usher, 1994; Diesmann, Gewaltig, \& Aertsen, 1996; Maršalek, Koch, \& Maunsell, 1997) showing which the ratio of the output jitter to the input jitter is consistently less than one and that it decreases for increasing numbers of inputs. Moreover, we identified the variation in the spike-generating thresholds of the neurons and the variation in the num- 
ber of active inputs as being important factors that determine the timing jitter in layered networks, in addition to the previously identified factors of axonal propagation times and synaptic jitter. This study of the synchronization problem using the techniques presented here is complementary to this study, since the problems addressed represent two extreme cases of the temporal information that the inputs contain: in the synchronization problem, all of the input arrival times are related, whereas for a Poisson distribution of PSP inputs, the arrival times are completely independent.

Details of the technique are presented in section 2 , and it is then applied to various neural models in section 3 . The result for the perfect integrator (Gerstein \& Mandelbrot, 1964), in which the decay of the membrane potential over time is neglected, is reproduced in section 3.1. The solution for the moments of the firing distribution in the Stein model (CIuss, 1967) is reproduced in section 3.2, and plots of the firing probability, mean interspike interval, with of the interspike interval distribution, and coefficient of variation are given. We also plot the output rate of firing as a function of the input rate for a number of thresholds. More general synaptic response functions, which include not only the decay of the membrane potential bat also its rise time, are considered in section 3.3. The effect of a distribution of PSP amplitudes and rates is examined in section 3.4, and the effect of including inhibitory PSPs is examined in section 3.5. The finat section contains a discussion of the results and the conclusions.

\section{Technique for Calculating Output Spike Density}

We calculate the interspike interval for an integrate-and-fire neuron with $N$ afferent fibers. The time of arrival of the inputs on the $k$ th fiber is modeled as a renewa: process, and the results in sections 2 . $i$ and 2.2 hold for any renewal process, that is, the successive time intervals between inputs are independent and identically distributed. The times of arrival of the inputs are described by the probability density function, which we denote by $p_{k}(t)$; this is the probability density of the interspike interval distribution on the $k^{\text {th }}$ fiber. In section 2.3 we examine the case in which the arrival times form a Poisson process with constant rate $\lambda$. However, the technique outlined here is applicable to probability densities other than the Poisson distribution, which is useful when refractory effects are considered and for nonstationary processes.

The membrane potentia: $V(t)$ is assumed to be reset to its initial value at time $t=0, V(0)=v_{0}$ after an action potential (AP) has been generated. The potential is the sum of the excitatory and inhibitory postsynaptic potentials (EISI's and IPSPS, respectively),

$$
V(t)=w_{n}+\sum_{k=1}^{N} a_{k} \sum_{m-i}^{x} u_{k}\left(t \cdots t_{k_{m}}\right) . \quad t \geq 0,
$$


where the index $k=1 \ldots N$ denotes the afferent fiber and the index $m$ denotes the $m$ th input from the particular fiber, whose time of arrival is $t_{k_{n}}$ $\left(0<t_{k}<t_{k_{2}}<\cdots<t_{k_{n}}<\cdots\right)$. The amplitude of the inputs from fiber $k$ is $a_{h}$ (positive for EPSPS and negative for IPSPS), and the time course of an input at the site of spike generation is described by the synaptic response function $u_{k}(t)$, which is zero for $t<0$ and positive for $t \geq 0$ with a peak value normalized to one (the definitions for the three cases examined here are given in equations 3.1,3.6, and 3.17). In section 3 we examine a number of synaptic response functrons corresponding to various neural models, including the perfect integrator model and the Stein model (also called the shot noise model). More general models of the synaptic response function enable us to model the time course of the incoming PSPs, taking into account both the leakage of the potential across the membrane and the effect of propagation along the dendritic tree.

The various versions of the integrate-and-fire neural model have as their key components a passive integration of the synaptic inputs, a voltage threshold for spike generation, and a lack of the specific currents that underlie spiking. The complex mechanisms by which the sodium and potassium conductances cause action potentials to be generated are not part of the model. An important assumption is also that the synaptic inputs interact only in a linear manner, so that phenomena involving nonlinear interaction of synaptic inputs, such as synaptic currents that depend on postsynaptic currents and pulse-paired facilitation and depression (which require higherorder terms in $u_{h}(t)$ ), are neglected. A recent review of the integrate-and-fire ncural model, giving details of the various variations of the model and comparisons with both experimental data and other models, is given in Koch, (1999).

In the results that follow, we express the relationship between the inputs and the threshold in terms of the threshold ratio $R$, which is defined as

$$
R: \frac{V_{\mathrm{th}:}-\vartheta_{\mathrm{a}}}{N a}=\frac{\theta}{N a} .
$$

where $N$ is the number of afferent fibers that contribute EPSPs and $a$ is the amplitude of the individual PSPS (taken here to all be equal in magnitude) and $\theta$ is the difference between the threshold potential, $V_{t h}$ and the resting potential, $v_{0}$. We choose the units of voltage to be set by the difference between the threshold and reset values, $\theta=V_{\text {th }}-v_{0}=1$. The utility of the parameterization of the threshold will become apparent when we consider how the results scale with increasing numbers of inputs, $N$, and decreasing amplitudes, $a$, in section 3 .

2.1 The Probability Density of the Potential. The probability density of the potential having the value $v$ at time $t$ is evaluated by considering the fraction of inputs for which this holds. This is given by the integral over the 
probability distribution of the inputs

$$
\begin{aligned}
p\left(v . t \mid \eta_{0}\right)=\prod_{k-1}^{x}\{ & \int_{0}^{x} d t_{k_{1}} p_{k}\left(t_{k_{1}}\right) \int_{t_{1}}^{x} d t_{k_{2}} p_{k}\left(t_{k_{2}}-t_{k_{1}}\right) \\
& \left.\times \int_{t_{k_{2}}}^{x} d t_{k_{3}} p_{k}\left(t_{k_{3}}-t_{k_{2}}\right) \ldots\right\} \delta(V(t)-v) .
\end{aligned}
$$

where $p\left(v . t \mid \nu_{0}\right)$ is the probability that the potential, $V(t)$, takes the value 0 at time $t$, given the initial condition $V(0)=v_{0}$. This initial condition corresponds to the reset of the membrane potential to $v_{0}$ immediately after the previous spike, which is assumed to occur at time $t=0$. The dots indicate the sequence of integrals, each corresponding to the successive arrival of inputs on the particular fiber (note that the integrals are normalized), and $\delta(x)$ is the Dirac delta function. Since the contributions from the afferent fibers are independent, the above probability density may be written as

$$
\begin{aligned}
p\left(v, t \mid v_{0}\right) & =\int_{-\infty}^{x} \frac{d x}{2 \pi} \exp \left\{i x\left(v-v_{0}\right)\right\} \prod_{k=1}^{N} F_{k}(x . t) \\
& =\int_{-\infty}^{x} \frac{d x}{2 \pi} \exp \left\{i x\left(v-v_{0}\right)+\sum_{k-1}^{N} \ln F_{k}(x, t)\right\}
\end{aligned}
$$

where the Fourier representation of the Dirac delta function is used:

$$
\delta(z)=\int_{-\infty}^{\infty} \frac{d x}{2 \pi} \exp \{-i x z\}
$$

The function $F_{k}(x, t)$ is given by

$$
\begin{aligned}
F_{k}(x, t)= & \left\{\int_{t}^{\infty} d t_{k_{1}} p_{k}\left(t_{k_{1}}\right) \int_{t_{k}}^{\infty} d t_{k_{2}} p_{k}\left(t_{k_{2}}-t_{k_{1}}\right) \ldots\right\} \\
& \times \exp \left\{-i x a_{k} \sum_{n_{t=1}}^{x} u\left(t-t_{k_{m}}\right)\right\}
\end{aligned}
$$

The exponential in the above expression for $F_{k}(x, t)$ can be expanded in terms of the individual amplitudes, $a_{k}$, and only the linear and quadratic terms are retained. A formal solution to the resulting equations is given (we do not address such questions as the convergence of the expansion). However, in order to obtain a handle on the accuracy of this approximation, the resuits are compared in the following sections with those obtained by 
numerical simulations. The expression for $F_{k}(x, t)$ gives

$$
\begin{aligned}
\ln F_{k}(x . t) & =\ln \left\{1-i x a_{k} D_{k}(t)-\frac{x^{2} a_{k}^{2}}{2} E_{k}(t)+O\left(a_{k}^{3}\right)\right\} \\
& =-i x a_{k} D_{k}(t)-\frac{x^{2} a_{k}^{2}}{2}\left(E_{k}(t)-D_{k}^{2}(t)\right)+O\left(a_{k}^{3}\right) .
\end{aligned}
$$

where $O(y)$ denotes a remander term that is smaller than $C \times\left|y^{3}\right|$ when $y$ lies in a neighborhood of 0 for some positive constant $C$. The functions $D_{k}(t)$ and $L_{h}(t)$ are given by

$$
\begin{aligned}
D_{k}(t)= & \left\{\int_{0}^{\infty} d t_{k_{1}} p_{k}\left(t_{k_{1}}\right) \int_{t_{k_{1}}}^{x} d t_{k_{2}} p_{k}\left(t_{k_{2}}-t_{k_{1}}\right) \ldots\right\} \sum_{m=1}^{\infty} u_{k}\left(t-t_{k_{m}}\right) \\
E_{-k}(t)= & \left\{\int_{0}^{x} d t_{k_{1}} p_{k}\left(t_{k_{1}}\right) \int_{i_{1}}^{x} d t_{k_{2}} p_{k}\left(t_{k_{2}}-t_{k_{1}}\right) \ldots\right\} \\
& \times \sum_{m: m^{\prime}=1}^{x} u_{k}\left(t-t_{k_{m}}\right) u_{k}\left(t-t_{k_{m}}\right) .
\end{aligned}
$$

The expressions for $D_{k}(t)$ and $E_{k}(t)$ may be solved using Laplace transforms, as shown in appendix $A$. Neglecting the $O\left(a_{k}^{3}\right)$ terms in equation 2.7 and retaining only the bnear and quadratic terms (the gaussian approximation), the probability density function can then be evaluated as

$$
\begin{aligned}
& p\left(r^{\prime}, t \mid v_{0}\right) \\
& =\int_{-x}^{\infty} \frac{d x}{2 \pi} \exp \left\{i x\left(v-v_{0}-\sum_{k}^{N} a_{k} D_{k}(t)\right)-\frac{x^{2}}{2} \sum_{k}^{N} a_{k}^{2}\left(E_{k}(t)-D_{k}^{2}(t)\right\}\right. \\
& =\frac{1}{\sqrt{2 \pi \Gamma(t)}} \exp \left\{-\frac{\left(v-v_{0}-\gamma(t)\right)^{2}}{2 \zeta(t)}\right\}
\end{aligned}
$$

with

$$
\begin{aligned}
& \Upsilon(t)=\sum_{k=1}^{N} a_{k} D_{k}(t) \\
& \Gamma(t)=\sum_{k=1}^{N} a_{k}^{2}\left(\Gamma_{k}(t)-D_{k}^{2}(t)\right) .
\end{aligned}
$$

In the case of the Poisson process, the expressions for $\Upsilon(t)$ and $I(t)$ take particularly simple forms, as will be shown in section 2.3 (We assume here 
that $\Gamma(t)>0$, which is indeed the case for Poisson inputs, as shown later in equation 2.23).

The gaussian approximation for $F_{k}(x, t)$, equation 2.7 (i.e., keeping only terms to second order in the amplitude $a_{k}$ of the individual postsynaptic contributions) is an approximation that is expected to wrork best for large values of $N$ and values of the amplitude that are small (voltage is measured in units of the difference $\theta$ between the threshold, $V_{\text {th }}$, and reset, $v_{0}$, potentials). We assume that the amplitude, $a_{k}$, scales with the number of inputs, $N$ (i.e., that the amplitude is of order $1 / N$ ) and that $\Upsilon(t)$ is therefore of the same order as $\theta$, thus giving a reasonable output rate of spikes. Consequently the threshold ratio, equation 2.2, is a useful quantity with which to compare neurons that sum different number of inputs of various amplitude. The results in the following sections do indeed support this assumption. 'The question of how many inputs and how small the individual synaptic amplitudes must be in order for the analysis to provide an accurate evaluation of the neural response will be addressed in subseguent sections, where we compare the results with those obtained from numerical simulations for a range of number of inputs and relative amplitudes. However, the situation in which a large number of small-amplitude postsynaptic potentials are required for the firing threshold to be reached is one that occurs in many neurons (Abeles, 1991; Douglas \& Martin, 1991).

We consider here the case in which the amplitudes of the contributions from the inputs are equal in magnitude, $\left|a_{k}\right|=a$, the rates on each of the incoming fibers are equal, $\lambda_{k}=\lambda$, and all of the inputs are excitatory. It is straightforward to generalize the results to any distribution of amplitudes, as discussed in section 3.4, since the amplitude dependence of the functions $\Upsilon(t) . \Gamma(t)$ is given explicitly in equation 2.10 (note that $D_{k}(t), E_{k}(t)$ are independent of the amplitude). In section 3.5 the generalization to the case in which there are both excitatory and inhibitory inputs is considered. Since there is no interaction from the contributions of different incoming fibers in this model, it is possible to consider having different synaptic response functions on different afferent fibers. This may enable us to model inputs coming from fibers that are situated or the dendritic tree at different distances from the site (typically the axon hillock) at which the summed potential generates the output spike. However, we consider here only the case in which the synaptic response function from each input is the same, $u_{k}(t)=u(t)$. Consequently all input fibers and their characteristics are equiralent, and therefore the index $k$ is dropped in the analysis of the following section.

2.2 First-Passage Time to Threshold. A spike is generated when the summed membrane potentia! reaches the threshold, $V_{\text {th }}\left(V_{\text {th }}=v_{0}+\theta\right)$, for the first time. The probability density of output spikes, which is the interspike interval (ISI) density, is therefore equivalent to the probability density of the first time that the potential, $V(t)$, reaches the threshold, which 
is called the first-passage time to threshoid density, denoted by $f_{t}(t)$. This may be obtained from the integral equation (Plesser \& Tanaka, 1997; Burkitt \& Clark, 1999a),

$$
p\left(v, t \mid v^{\prime}(0)=\int_{0}^{t} d t^{\prime} f_{t}\left(t^{\prime}\right) p\left(v^{\prime}, t \mid V_{\mathrm{th},}, t^{\prime} . v_{0}\right), \quad v \geq V_{\mathrm{th}}\right.
$$

where $\hat{p}\left(v_{2} . t_{2} \mid v_{1}, t_{1} . v_{0}\right)$ is an approximation to the conditional probability density of the potential. The conditional probability density, $p\left(v_{2}, t_{2}\right)$ $\left.v_{1}, t_{1}, v_{0}\right)$, is defined as the probability that the potential has the value $v_{2}$ at time $t_{2}$. given that $V\left(t_{1}\right)=v_{1}$ and the reset value of the potential after a spike is $\%$. Our approximation to the conditional probability density, $\hat{p}\left(v_{2}, t_{2} \mid v_{1}, t_{1}, v_{0}\right)$, has the same definition, with the added restriction that the potential is subthreshold in the time interval $0 \leq t<t^{\prime}$. If the fluctuations in the input currents are not temporally correlated, then the approximate expression is exact, as is the case for both the perfect integrator model and the Stein model with Poisson distributed inputs. However, the two expressions will differ if there are temporal correlations, which occurs if the presynaptic spikes have correlations or the synaptic currents have a finite duration.

The integral expression for $f_{H}(i)$ given above is analogous to the renewal equation (Tuckwell, 1988b),

$$
p\left(v, t \mid v_{0}\right)=\int_{0}^{t} d t^{\prime} f_{\theta}\left(t^{\prime}\right) p\left(v, t-t^{\prime} \mid V_{\mathrm{th}}\right)
$$

'This renewal equation is a special case of equation 2.11 that is valid only for the perfect integrator (i.e., nonleaky) neural model, and it arises because the perfect integrator relates the conditional probability density to the original probability density, that is, $p\left(\tau, t \mid V_{\mathrm{th}}, t^{\prime}, v_{0}\right)=p\left(\tau, t-t^{\prime}{ }_{\mathrm{th}}\right)$ for $\tau^{\prime} \geq V_{\mathrm{t}}$ (in more general neural models, there is no such spatial and temporal homogeneity) There is also no assumption about the stationarity of the conditional probability density, $\hat{p}\left(v, t \mid V_{\mathrm{th}} . t^{\prime}, v_{0}\right)$, in equation 2.11 , it does not have any time-translational invariance. The conditional probabilsty density takes account of possible multiple crossings of the threshold between times $t^{\prime}$ and $t$.

The conditional probability density is given by (Bayes' theorem)

$$
p\left(\tau_{2}, t_{2} \mid v_{1}, t_{1}, v_{0}\right)=\frac{p\left(\tau_{2}, t_{2}, \tau_{1}, t_{1} \mid v_{0}\right)}{p\left(v_{1}, t_{1} \mid v_{0}\right)}
$$

and the joint probability density $p\left(\tau_{2}, t_{2}, v_{1}, t_{1} \mid \tau_{0}\right)$, which is the probability that $V(t)$ takes the value $z_{1}$ at time $t_{1}$ and takes the value $v_{2}$ at time $t_{2}$ (given that the reset value of the potential after a spike is generated is again at $\tau_{0}$ ), 
may be evaluated in a similar way to the probability density, equations 2.3 and 2.4 :

$$
\begin{aligned}
& p\left(v_{2}, t_{2}, v_{1}, t_{1} \mid v_{0}\right\} \\
& =\prod_{k=1}^{N}\left\{\int_{0}^{x} d t_{k_{1}} p_{k}\left(t_{k_{2}}\right) \int_{t_{k_{1}}}^{x} d t_{k_{2}} p_{k}\left(t_{k_{2}}-t_{k_{2}}\right) \ldots\right\} \delta\left(V\left(t_{1}\right)-v_{1}\right) \delta\left(V\left(t_{2}\right)-v_{2}\right) \\
& =\int_{x}^{x} \frac{d x_{2}}{2 \pi} \int_{-\infty}^{x} \frac{d x_{1}}{2 \pi} \exp \left\{i x_{2}\left(v_{2}-v_{0}\right)+i x_{1}\left(v_{1}-v_{0}\right)\right. \\
& \left.+N \ln F\left(x_{2}, x_{1}, t_{2}, t_{1}\right)\right\} .
\end{aligned}
$$

The function $\ln F\left(x_{2}, x_{1}, t_{2}, t_{1}\right)$ is expanded in the amplitude $a$ of the synaptic response function as before (see equation 2.7 ) and contains cross-terms in $x_{2} x_{1}$ :

$$
\begin{aligned}
\ln F\left(x_{2}, x_{i}, t_{2}, t_{1}\right) & \\
=\ln & \left\{1-i x_{2} a D\left(t_{2}\right)-i x_{1} a D\left(t_{1}\right)\right. \\
& \left.\quad-\frac{x_{2}^{2}}{2} a^{2} E\left(t_{2}\right)-\frac{x_{1}^{2}}{2} a^{2} E\left(t_{1}\right)-x_{2} x_{1} a^{2} G\left(t_{2}, t_{1}\right)+O\left(a^{3}\right)\right\} \\
= & -i x_{2} a D\left(t_{2}\right)-i x_{1} a D\left(t_{1}\right) \\
& \quad-\frac{x_{2}^{2}}{2} a^{2}\left(E\left(t_{2}\right)-D^{2}\left(t_{2}\right)\right)-\frac{x_{1}^{2}}{2} a^{2}\left(E\left(t_{1}\right)-D^{2}\left(t_{1}\right)\right) \\
& -x_{2} x_{1} a^{2}\left(G\left(t_{2}, t_{1}\right)-D\left(t_{2}\right) D\left(t_{1}\right)\right)+O\left(a^{3}\right),
\end{aligned}
$$

where $D(t), E(t)$ are given by equation 2.8 (the index $k$ is dropped, since all incoming fibers are treated identically) and $G\left(t_{2}, t_{1}\right)$ is given by

$$
\begin{aligned}
G\left(t_{2}, t_{1}\right)= & \left\{\int_{0}^{\infty} d t_{1}^{\prime} p\left(t_{i}^{\prime}\right) \int_{t_{1}^{\prime}}^{\infty} d t_{2}^{\prime} p\left(t_{2}^{\prime}-t_{1}^{\prime}\right) \ldots\right\} \\
& \times \sum_{m=1}^{\infty} u\left(t_{1}-t_{m}^{\prime}\right) \sum_{m^{\prime}=1}^{\infty} u\left(t_{2}-t_{m}^{\prime}\right) .
\end{aligned}
$$

This expression for $G\left(t_{2}, t_{1}\right)$ may also be solved in terms of Laplace transforms (see appendix $B$ ).

Once again we retain only the linear and quadratic terms in the amplitude, $a$, in equation 2.14 . In order to evaluate the integrals in equation 2.14 we make a change of variable $y_{1}=x_{1}+\kappa\left(t_{2}, t_{1}\right) x_{2}$ where $\kappa\left(t_{2}, t_{1}\right)$ is chosen 
in order to eliminate the cross-term $x_{2} y_{1}$ :

$$
\kappa\left(t_{2}, t_{1}\right)=\frac{N a^{2}\left[G\left(t_{2} \cdot t_{1}\right)-D\left(t_{2}\right) D\left(t_{1}\right)\right]}{\Gamma\left(t_{1}\right)} .
$$

The $y_{1}$ and $x_{2}$ integrals are now independent and may be evaluated. The $y_{1}$ integral yields exactly $p\left(v_{1}, t_{1} \mid v_{0}\right)$, and the $x_{2}$ integral gives the conditional probability density.

$$
\begin{aligned}
p\left(v_{2}, t_{2}\right. & v_{1}, t_{1}, v_{(1)} \\
= & \frac{1}{\sqrt{2 \pi \gamma\left(t_{2}, t_{1}\right)}} \\
& \times \exp \left\{-\frac{\left[\left(v_{2}-v_{0}-\Upsilon\left(t_{2}\right) \cdot \kappa\left(t_{2}, t_{1}\right)\left(v_{1}-v_{0}-\Upsilon\left(t_{1}\right)\right)\right\}^{2}\right.}{2 \gamma\left(t_{2}, t_{1}\right)}\right\},
\end{aligned}
$$

where

$$
\gamma\left(t_{2}, t_{1}\right)=\Gamma\left(t_{2}\right)-\kappa^{2}\left(t_{2}, t_{1}\right) \Gamma\left(t_{1}\right)
$$

The value of $\gamma\left(t_{2}, t_{1}\right)$ is guaranteed to be positive (for $\left.t_{2}>t_{1}\right)$ for Poissondistributed inputs isee the end of section 2.3).

The first passage-time density is evaluated using equation 2.11. For inputs described by a Poisson process, the equation may be solved explicitly for two neural models: the perfect integrator and the Stein model. In the case of the perfect integrator, the method reproduces the known result of the inverse gaussian density (Tuckwell, 1988b). as discussed in section 3.1. In the case of the Stein model, this technique reproduces the known solution (Gluss, 1967), in which the moments of the first passage-time density are expressed in terms of the Laplace transforms of the probability density and the conditional probability density (section 3.2). However, apart from these special cases, equation 2.11 has not been solved analytically for $f_{s}(t)$.

We solve equation 2.11 for $f_{\theta}(t)$ numerically using standard methods for Volterra integral equations (Press, Flannery, Teukolsky, \& Vetterling, 1992; Plesser \& Tanaka, 1997). The moments of this distribution are then straightforward to evaluate. The zeroth moment of the distribution is the integral of the probability density itself and gives the probability $p$ of a spike being produced. The first moment, $t_{f}$ is the average time of the first threshold crossing and hence the ISI. The second moment $\sigma_{\text {out }}$ is the spread of the distribution in time of the output spikes (i.e., the width of the ISI). Although the ISI distributions are frequently characterized by their moments, the numerical results show clearly that they are somewhat skewed, resembling more the generalized inverse gaussian distribution (lyengar \& Liao, 1997). 
2.3 Poisson Process. In a renewal process, the interarrival times of the presynaptic inputs are independent and identically distributed with an arbitrary distribution. The Poisson process is a particular case of a renewal process in which the distribution is exponential; the probability density of the first input on fiber $k$ is

$$
p_{k}\left(t_{k}\right)=\lambda_{k} e^{-\lambda_{k} t_{k}}
$$

where $t_{k}$ is the time since the previous input and $\lambda_{k}$ is the constant rate of input on the $k$ th fiber. Likewise, the probability density of the $m$ th input on fiber $k$ is

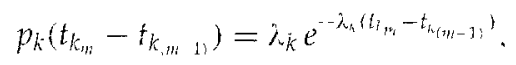

where $t_{k_{n-1}}$, is the time of the preceding input (i.e., the $(m-1)$ th input). The Laplace transform of $p_{k}(t)$ is

$$
p_{k, L}(s)=\frac{\lambda_{k}}{s+\lambda_{k}}
$$

and $D_{k, L}(s), F_{k . I}(s)$, equations $A .1$ and $\Lambda .2$, may be evaluated to yield the functions $\Upsilon(t)$ and $\Gamma(t)$, equation 2.10 ,

$$
\begin{array}{ll}
\Upsilon(t)=\sum_{k} a_{k} \lambda_{k} \int_{0}^{t} d t^{\prime} u\left(t^{\prime}\right) & \stackrel{\left(\lambda_{k}=\lambda\right)}{=} N a \lambda \int_{0}^{t} d t^{\prime} u\left(t^{\prime}\right) \\
\Gamma(t)=\sum_{k} a_{k}^{2} \lambda_{k} \int_{0}^{1} a t^{\prime} u^{2}\left(t^{\prime}\right) & \stackrel{\left(\lambda_{k}=\lambda\right)}{=} N a^{2} \lambda \int_{0}^{t} d t^{\prime} u t^{2}\left(t^{\prime}\right) .
\end{array}
$$

For a Poisson process, these expressions may also be arrived at by noting that a superposition of Poisson processes gives a Poisson process (with a rate that is the sum of the contributing rates) and by integrating the synaptic response function to obtain the average and variance of the depolarization (Stein, 1967; Abeles, 1982b).

The conditional probability density, $p\left(v_{2}, t_{2} \mid v_{3}, t_{1}, v_{n}\right)$, may be obtained in closed analytic form for any synaptic response function, $u(t)$, since the expression for $\kappa\left(t_{2}, t_{1}\right.$, follows from equation 2.17 and the solution of $G\left(t_{2}, t_{1}\right)$ in appendix $B$, equation $B .1$ :

$$
\kappa\left(t_{2}, t_{1}\right)=\frac{N a^{2} \lambda \int_{0}^{t_{1}} d t^{\prime} u\left(t^{\prime}\right) u\left(\Delta+t^{\prime}\right)}{\Gamma\left(t_{1}\right)} . \quad \Delta=t_{2}-t_{1} \geq 0 .
$$

Note that the rate, $\lambda$, given here is the average rate on each afferent fiber, and therefore the total rate of inputs is $N \lambda$. For a Poisson process, $\kappa\left(t_{2}, t_{1}\right)$ and 
$\Gamma\left(t_{1}\right)$ are related to the autocovariance of the sequence of Poisson inputs, $C\left(t_{2}, t_{1}\right)$, by the relationship (Papoulis, 1991),

$$
\kappa\left(t_{2}, t_{1}\right)=\frac{C\left(t_{2}, t_{1}\right)}{C\left(t_{3}, t_{1}\right)}, \quad I\left(t_{1}\right)=C\left(t_{1}, t_{1}\right)
$$

which ensures that the expression for $\gamma\left(t_{2} . t_{1}\right)$, equation 2.19 , is positive definite.

\section{Neural Models}

3.1 Perfect Integrator Model. The perfect integrator model (also called the leakless integrate-and-fire model) is the simplest of the neural models to analyze, since there is no decay of the potential with time. Although this is clearly an unrealistic assumption, it provides a reasonable approximation for situations in which the integration occurs over a much shorter timescale than the decay constant. The synaptic response function for the perfect integrator simply takes the form

$$
u(t)= \begin{cases}1 & \text { for } t \geq 0 \\ 0 & \text { for } t<0\end{cases}
$$

The probability density of the potential for this model is well known (Tuckwell, 1988b) and may be evaluated as a Wiener process with drift (Gerstein \& Mandelbrot, 1964). The resulting expressions are identical to those obtained using the above technique. The expressions for $\Upsilon(t)$ and $\Gamma(t)$, for the case in which all the inputs are excitatory and the input rate on each fiber is identical, follow from equation 2.23:

$$
\begin{aligned}
& \Upsilon(t)=N a \lambda t \\
& \Gamma(t)=N a^{2} i t
\end{aligned}
$$

Moreover, the expressions for $k\left(t_{2}, t_{1}\right)$ and $\gamma\left(t_{2}, t_{1}\right)$ are

$$
\begin{aligned}
& \kappa\left(t_{2}, t_{1}\right)=1 \\
& \gamma\left(t_{2}, t_{1}\right)=\Gamma\left(t_{2}\right)-\Upsilon\left(t_{1}\right)=N a^{2} \lambda\left(t_{2}-t_{1}\right),
\end{aligned}
$$

and therefore the conditional probability density is related to the probability density in this particular case by

$$
p\left(v_{2} \cdot t_{2} \mid v_{1}, t_{1} \cdot v_{0}\right)=p\left(v_{2}, t_{2}-t_{3} \mid v_{1}\right) . \quad v_{2}>v_{1} .
$$

Equation 2.11 is therefore a genuine renewal equation, as we would expect for the perfect integrator. The density of the output spike distribution is 
given by standard Laplace transform techniques as the inverse gaussian density (Gerstein \& Mandelbrot, 1964),

$$
f_{G}(t)=\frac{\theta}{\sqrt{2 \pi N a^{2} \lambda t^{3}}} \exp \left\{-\frac{(\theta-N a \lambda t)^{2}}{2 N a^{2} \lambda t}\right\}
$$

where the threshold is given by $V_{\text {th }}=v_{0}+\theta$.

3.2 Solution of the Stein Model. The passive decay of the membrane potential may be incorporated in the model by a synaptic response function of the form (Stein, 1965),

$$
u(t)= \begin{cases}e^{-t / \tau} & \text { for } t \geq 0 \\ 0 & \text { for } t<0\end{cases}
$$

where $\tau$ is the time constant of the membrane potential decay. This form of the integrate-and-fire model with stochastic inputs was first studied by Stein (1965) and is known as the Stein model or shot-noise threshold model. For a Poisson process in the case in which all the inputs are excitatory (inhibitory inputs are considered in section 3.5 ) and the input rate on each fiber is identical, it is straightforward, using equations 2.19, 2.23, and 2.24, to calculate the functions

$$
\begin{aligned}
& \Upsilon(t)=N a \lambda \tau\left(1-e^{-t_{i} \tau}\right) \\
& \Gamma(t)=\frac{1}{2} N a^{2} \lambda \tau\left(1-e^{2 t / \tau}\right) \\
& \kappa\left(t_{2}, t_{1}\right)=c^{-\left(t_{2}-t_{i}\right) / \tau} \\
& \gamma\left(t_{2}, t_{1}\right)=\frac{1}{2} N a^{2} \lambda \tau\left(1-e^{-2\left(t_{2}-t_{1}\right) / \tau}\right)=\Gamma\left(t_{2}-t_{1}\right) .
\end{aligned}
$$

Thus for the Stein model the conditional probability density, equation 3.19, possesses temporal homogeneity (i.e., it is stationary),

$$
p\left(v_{2}, t_{2} \mid v_{1}, t_{3}, v_{0}\right) \equiv q\left(t_{2}-t_{1}: v_{2}, v_{1}, v_{0}\right),
$$

where the function $q\left(t: v_{2}, v_{1}, v_{0}\right)$, which is introduce 3 here to make clearer the equivalence with the known result (Gluss, 1967), is

$$
\begin{aligned}
q\left(t ; v_{2} \cdot v_{1}, v_{0}\right)= & \frac{1}{\sqrt{2 \pi \Gamma(t)}} \\
& \times \exp \left\{\frac{\left[v_{2}-v_{1} e^{-t / \tau}-v_{0}\left(1-t^{-t / \tau}\right)-\Upsilon(t)\right]^{2}}{2 \Gamma(t)}\right\} .
\end{aligned}
$$


The equation for the probability density of the output spike distribution, equation 2.11, is therefore a convolution equation that allows a solution in terms of Laplace transforms

$$
f_{f I}(s)=\frac{p_{I}\left(\tau, s \mid v_{0}\right)}{q_{L}\left(s ; v \cdot V_{\mathrm{th}} \cdot v_{0}\right)}=\frac{q_{L}\left(s ; v, v_{0}, v_{n}\right)}{q_{L}\left(s ; v, V_{\mathrm{th}_{\mathrm{L}}}, v_{0}\right)}
$$

where we have used the following straightforward general identity between the probability density and conditional probability density,

$$
p\left(v, t \mid v_{0}\right)=p\left(v_{,} t \mid v_{0}, 0, v_{0}\right)=q\left(t ; v^{\prime}, v_{0}, v_{0}\right) .
$$

This result for $f_{\theta i}(s)$, equation 3.10 , valid for $v \geq V_{\text {th }}$ agrees exactly with the known result obtained for $f_{t L}(s)$ in the case $v=V_{\text {th }}$ in the diffusion limit (Gluss, 1967). The first three moments of the probability density $f_{t}(t)$ may be obtained from the Laplace transform by the relationships

$$
\begin{aligned}
& \int_{0}^{\chi} d t f_{t}(t)=f_{t I I}(0) \\
& \int_{0}^{x} d t t f_{\theta}(t)=-\left(\frac{d f_{\theta L}(s)}{d s}\right)_{s-0} \\
& \int_{0}^{x} d t t^{2} f_{\theta}(t)=\left(\frac{d^{2} f_{H L}(s)}{d s^{2}}\right)_{s=0} .
\end{aligned}
$$

Denoting derivatives with respect to s by a prime and taking the value of cach of the functions at $s=0$, and with values of $v \geq V_{\text {th }}$, we obtain solutions for $\rho$, the probability of a spike's being generated in a finite time interval, $t_{t}$, the mean ISI, and $\sigma_{\text {out }}$, the standard deviation of the output distribution of spikes (the width of the ISI distribution),

$$
\begin{aligned}
& \rho=\int_{0}^{\alpha} d t f_{\theta}(t)=\frac{p_{L}}{q_{L}} \\
& t_{i}=\frac{1}{\rho} \int_{0}^{x} d t t f_{\theta}(t)=\frac{q_{L_{L}}^{\prime}}{q_{L}}-\frac{p_{L}^{\prime}}{p_{L}} \\
& \sigma_{\text {out }}=\frac{1}{\rho} \int_{0}^{x} d t\left(t \cdots t_{f}\right)^{2} f_{\theta}(t)=\frac{p_{L}^{\prime \prime}}{p_{L}}-\frac{q_{L}^{\prime \prime}}{q_{L}}+\frac{q_{L}^{\prime 2}}{q_{L}^{2}}-\frac{p_{L}^{\prime 2}}{p_{L}^{2}},
\end{aligned}
$$

where the primes denote derivatives with respect to $s$ and

$$
p_{L}=p_{L}\left(\tau, 0 \mid v_{0}\right) . \quad q_{L} \equiv q_{L}\left(0 ; v, V_{\text {th }}, v_{0}\right)
$$

The evaluation of the expressions for $p_{L}^{\prime}, q_{l}^{\prime}, p_{l}^{\prime \prime}$, and $q_{L}^{\prime \prime}$ as numerical integrals follows from the standard properties of Laplace transforms, as in equation 3.12 . 
While it is perfectly possible to evaluate the parameters $\rho, t_{f}$ and $\sigma_{\text {out }}$ numerically using the above Laplace transform methods, wre have used direct numerical methods for Volterra integral equations (Press et al., 1992; Plesser \& Tanaka, 1997) to evaluate the first-passage time density fo $(t)$ from its definition in equation 2.11 using equation 2.19. This equation has been solved for some typical parameter values in order to illustrate the nature of the solutions and comparc them with results in subsequent sections, where we include synaptic response functions with finite rise times (section 3.3) and inhibition (section 3.5).

A typical plot of the firing probability, $\rho$ (see equation 3.13), as a function of the threshold ratio, $R$ (see equation 2.2), is given in Figure $\$ for various numbers of afferent fibers, $N$. The input rate of EPSPs on each of the incoming fibers is $i_{m}=1.0$, and time is measured in units of the membrane time constant, $\tau=1.0$ (rates are given as spikes per unit of $\tau$ ). The curves represent interpolations between solutions that were evaluated at intervals of 0.01 on the $R$-axis. The steepest curve corresponds to the largest number of inputs $(N=1024)$ and indicates an abrupt change between a firing regime at low threshold ratios and a guiescent (nontiring) regime at high threshold ratios. In contrast, where there are tewer inputs (smaller $N$ values), this transition between firing and nonfiring regimes extends over a broader range of threshold ratios, $R$.

The value of the threshold ratio at which this transition from firing to nonfiring behavior takes place we define as the critical threshold ratio, $R_{\text {crit }}$. The criteria used to define the critical threshold ratio in this plot were that the probability of firing, $p$, be cqual to 0.05 , which was chosen to signify that the probability had risen significantly above zero (in principle, the criteria could be set at any intermediate value between zero and one). The dependence of the critical threshold ratio on the rate of inputs $\lambda_{1 n}$ is shown in Figure 2, where it is plotted for inputs $N=16,32,64,128,256,512,1024$, and the results for equal input rates, $\lambda_{1 n}$, are connected by lines. The results indicate that higher rates of input will generate an output spike over a larger range of threshold ratios, as expected. Moreover, the plots in Figure 2 show that the critical threshold ratio, $R_{\text {crit }}$, decreases only slightly as the number of inputs, $N$, increases. Consequently a neuron with a small number of large-amplitude inputs has a similar threshold ratio to one with a large number of small-amplitude inputs. In the large- $N$ limit, the behavior becomes deterministic as the variance of the inputs decreases $\left(\lim _{(N \rightarrow x)} \Gamma(t)=0\right)$, and consequently a spike output is produced when the mean input reaches the threshold. The critical threshold ratio is therefore given by the condition $\lim _{\left(\lambda^{2}-\rightarrow \infty\right.} \Upsilon \Upsilon(t \rightarrow \infty)=\theta$, which gives a value of $\lim _{(N \rightarrow \infty)} R_{\mathrm{crit}}=\tau \lambda_{\text {In }}$ (from the first expression in equation 3.7), consistent with the finite- $N$ results in Figure 2 (for which $\tau=1$ ).

The mean ISI, $t_{\text {f }}$ (equation 3.13), is plotted in Figure 3 for a number of input rates, $i_{1 n}$ (the lines represent interpolations between closely spaced indivalual numerical solutions). The solid lines are the results for $N=$ 


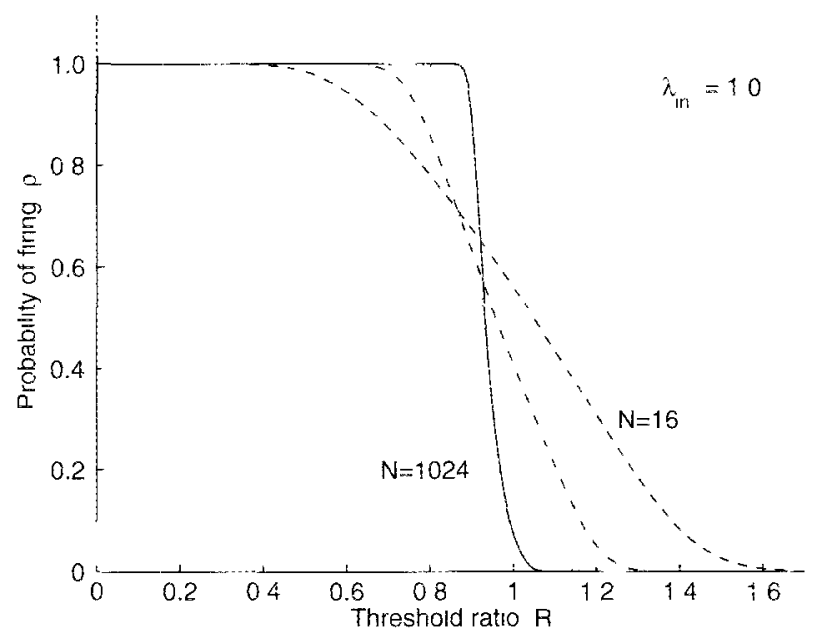

Figure 1: Plots of the probability of firing, $p$ (equation 3.13), versus threshold ratio, $R$ (equation 2.2), for the Stein model. The rate of incoming FPSPs is $\lambda_{1 n}=1.0$ on each afferent fiber, with time in units of the membrane time constant, $\tau$ (i.e., rates given as spikes per unit of $\tau$ ), and number of inputs $N=1024$ (solid line), 256 (dotted line), 64 (dash-dotted line), and 16 (dashed line).

1024 inputs, which indicate that for all threshold ratios, $R$, the mean $I S I, t_{f}$, decreases as the input rate, $\lambda_{1}$, increases. Likewise. lower threshold ratios, $R$, cause shorter mean ISIs, $t_{f}$, for any fixed input rate, $\dot{\lambda}_{\mathrm{in}}$. The results have only a very slight dependence on the number of inputs, as illustrated by the dashdotted line for $N=16$ inputs at $\lambda_{1 n}=1.5$, which is almost indistinguishable from the $N=1024$ results at the same input rate. The dotted lines give the values of the threshold ratio, $R$, where the rate of the incoming PSPs, $\lambda_{1 n}$, equals the rate of the output spikes, $\lambda_{\text {out }}=1 / t_{f}$, which they generate. In general we expect that in the normal operating regime of a network of such neurons, there would indeed be some approximate equality between the input and output rates, in order for the neural information to be propagated to further stages of processing.

The relationship between the input rate, $\lambda_{\mathrm{m}}$, and the output rate, $\lambda_{\text {out }}$, is illustrated in Figure 4 (joined by solid lines) for the Stein model with $N=1024$ inputs and three values of the threshold ratio, $R$ (the dashed lines connect results for the synaptic response function discussed in section 3.3). This relationship for the Stein model is substantially linear, with lower threshold ratios, $R$, resulting in higher output rates, $\lambda_{\text {out }}$. Note that refractory effects, which result in a saturation of the output rate, have not been included in this plot. 


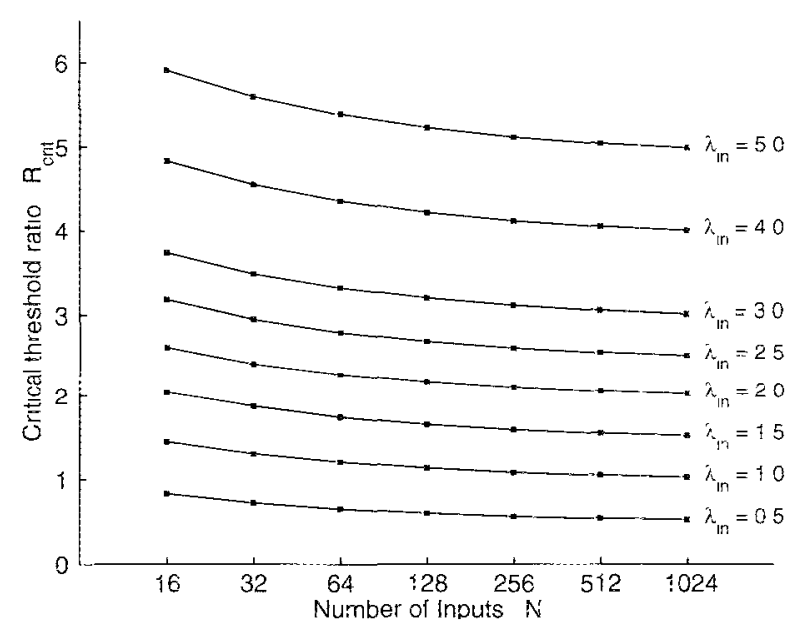

Figure 2: Dependence of the critical threshold ratio, $K_{\text {crit }}$, on the number of inputs, $N$, and the rate of inputs, $\lambda$, for the Stein model (with units $\tau-1.0) . R_{\text {cr.t }}$ was defined as the threshold ratio, $R$, above which the firing probability, $f$, feli below 0.05 .

The width of the ISI distribution. $\sigma_{\text {out }}$ (equation 3.13), as a function of the input rate, $\lambda_{\mathrm{in}}$, is illustrated in Figure 5 for a threshold ratio value of $R=0.5$, input rates in the range $0.5 \leq \dot{\lambda}_{1 n} \leq 2.0$ and various numbers of inputs, $N$. Also plotted is the coefficient of variation of the ISI distribution,

$$
C V_{\mathrm{ISi}}=\frac{\sigma_{\text {out }}}{t_{f}}=\sigma_{\text {out }} \lambda_{\text {out }}
$$

The lower value of $\lambda_{1 n}$ on each of the lines in this plot is determined by the critical threshold ratio $R_{\text {crit }}$.

The error bars joined by dotted lines represent the results, with their statistical errors, of numerical simulations involving 10,000 trials for values of $N=16,32$, and 64 . A stream of Poisson-distributed inputs was generated using a pseudorandom number generator, and the crror bars, which represent the statistical error, are only barely discernible in this plot at large values of $\lambda_{1 n}$, since the agreement between simulation and analytical results is so close. Near the critical threshold, the error bars become larger, and there is a small difference for the smaller values of $N$ in the results for $C V$ between the simulations and the analytical results given here.

These results indicate that the width of the output spike distribution, $\sigma_{\text {otit, }}$ decreases with both increasing number of inputs, $N$, and increasing rate of inputs, $\lambda_{1 n}$. The coefficient of variation behaves in much the same 


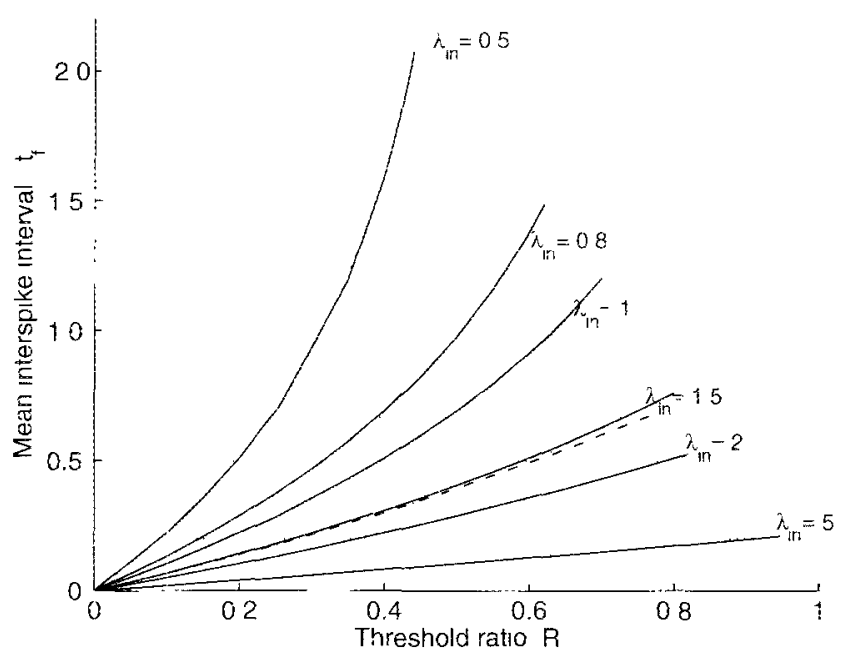

Figure 3: Dependence of the mean ISI, $t_{f}$, on the threshold ratio, $K$, for the Stein model for $N=1024$ (solid lines) with various rates of firing $\lambda_{1 ;}$. Also plotted are results for $N=16$ with $\lambda_{1:}=1.5$ (dash-dotted line). The dotted lines indicate the values of the threshold ratio, $R$, at which the input rate; $\lambda_{i n}$, is equal to the output rate, $\lambda_{\text {out }}$. Time is measured in units of the membrane time constant, $\tau$ (i.e., rates are given as spikes per unit of $\tau$ ).

way for values of the input rate above the critical threshold; that is, $C V$ decreases as $N$ increases in this domain. However, in the neighborhood of the critical threshold (i.e., $\lambda_{1 n}<0.5$ ), the coefficient of variation increases $s u b-$ stantially. These results indicate that there are two quite different regimes: (1) above the critical threshold, $\tau \lambda_{\text {in }}>R_{\text {crut }}$ and (2) near the critical threshold, $\tau \lambda_{\text {n }} \approx R_{\text {crit }}$. In the region substantially above the critical threshold, the coefficient of variation decreases with increasing $N$ in the way observed in Softky and Koch (1993), which led them to conclude that there was a conflict between the values of $C V$ observed experimentally and the results of the integrate-and-fire model. However, in the region near the critical threshold, the coefficient of variation is substantially larger and well within the range of experimental observations (Softky \& Koch, 1992; Shadlen \& Newsome, 1998). Softky and Koch (1992) analyzed recordings from the primary visual cortex and extrastriate cortex of awake, behaving macaque monkeys and found values of $\mathrm{CV}$ in the range $0.5-1.0$. Shadlen and Newsome (1998) found somew hat higher values of $C V$ in the range $1.0-1.5$ in their recordings from the visual cortex of alert, behaving rhesus monkeys. However, Gur, Beylin, and Snodderly (1997) found that a large component of the response vari- 


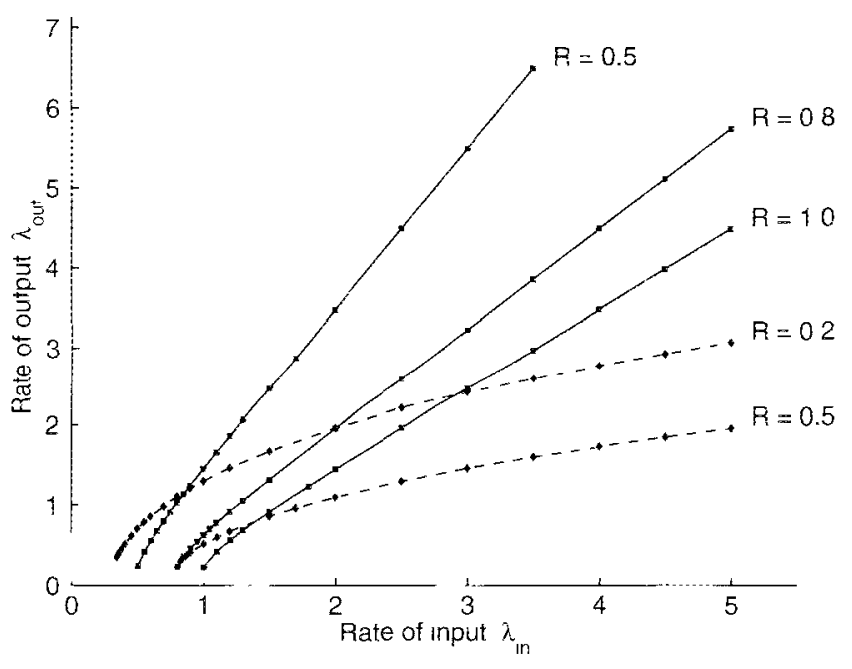

Figure 4: Mean output rate, $\lambda_{\text {out }}$, of spikes generated as a function of the input rate, $\lambda_{3 n}$, of afferent PSPS for $N=1024$ inputs. The solid lines are the results for the Stein model with the three threshold ratios given. Also plotted in dashed ines are the results for the alpha model (equation 3.17) with the two threshold ratios given. Time is measured in units of the membrane time constant, $\tau$ (i.e., rates are given as spikes per unit of $\tau$ ).

ability in alert monkeys was caused by fixational eye movements, and that when this was controlled for, the resulting values of $C V$ were found to be in the range $0.1-0.5$, with a systematic effect for stimulus strength (the variance was independent of the stimulus strength, whereas the output spike rate increased as the stimulus strength increased, resulting in lower values of $(V)$.

Shadlen and Newsome (1998) simulated three models for the integration of synaptic input, which in the units used here correspond to (1) membrane time constant $\tau=20 \mathrm{~ms}$, no inhibition, excitatory inputs with a rate $\lambda_{\mathrm{nn}}=50$ spikes $/ \mathrm{sec}=1.0$ spikes $/ \tau, N=300$ inputs, and amplitude $a=1 / 150$, giving a threshold ratio of $R=0.5$, and an output rate of $\lambda$ out $=50$ spikes $/ \mathrm{sec}=1.0$ spikes $\tau$; (2) membrane time constant $\tau=1 \mathrm{~ms}$, no inhibition, excitatory inputs with a rate; $\lambda_{1 \mathrm{n}}=50$ spikes $/ \mathrm{sec}=0.05$ spikes $/ \tau, N=300$ inputs, and amplitude $a=1 / 16$, giving a threshold ratio of $R=16 / 300 \approx 0.05$, and an output rate of $\lambda_{\text {out }}=50$ spikes $/ \mathrm{sec}=0.05$ spikes $/ \tau$; and (3) membrane time constant $\tau=20 \mathrm{~ms}$, balanced excitatory and inhibitory inputs with input

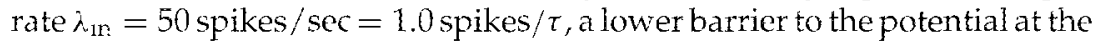
reset potential, $N=300$ inputs, amplitude $a=1 / 15$, giving a threshold ratio 
of $R=0.05$, and an output rate of $\lambda_{\text {out }}=50$ spikes $/ \mathrm{sec}=1.0$ spikes $/ \tau$. Their results for case (1) correspond to the value of the threshold ratio, $R=0.5$, plotted in Figure 5, and the results plotted in this figure are consistent with their results and extend them to a whole range of input rates and numbers of inputs (their results are for $\lambda_{1 n}=1.0$ spikes $/ \tau, N=300$ ). These results and their implications are discussed further when we look at the effect of including inhibitory inputs in section 3.5 .

3.3 Postsynaptic Response Functions with Finite Rise Times. Both the perfect integrator model and the Stein model analyzed above have discontinuous voltage trajectories in which an arriving EPSI causes an instantaneous jump of amplitude $a$ in the voltage. There are a number of models of the time course of EPSI's that have a smoothly varying voltage similar to that observed in intracellular recordings (Rhode \& Smith, 1986; Paolini, Clark, \& Burkitt, 1997). One such model is that in which the synaptic input current has a time course given by the alpha function (Jack, Noble, \& Tsien, $1985)$,

$$
I(t)=k t e^{\alpha i}, \quad \alpha>0,
$$

which corresponds to delivering a total charge of $k / \alpha^{2}$ to the cell. The synaptic response function $u(t)$ of this model (which we call the alpha model) may be evaluated by integrating the voltage in the equivalent circuit representation of the integrate-and-fire neural model. The resulting expression, given $u(0)=0$, is (Tuckwel, 1988a),

$$
m(t)= \begin{cases}\frac{k e^{-1, \tau}}{B C}\left[t e^{B, t}-\frac{\left(e^{B t}-1\right)}{B}\right], & B \neq 0, \quad t \geq 0 \\ \frac{k t^{2}}{2 C} e^{-t_{i} / \tau} . & B=0, \quad t \geq 0 \\ 0 . & t<0 .\end{cases}
$$

where $\tau=R C$ and $B=1 / \tau-\alpha$. The Stein model may be recovered in the limit $\alpha \rightarrow \infty$. Models such as this, which have a finite rise time, provide an approximation of the postsynaptic potential at the soma (or specifically at the site at which the action potential is generated, typically the axon hillock) that incorporates the time courst of the diffusion of the current along the dendritic tree (Tuckwell, 1988a).

The equation for the density of output spikes, $f_{H}(t)$ (see equation 2.11), may be solved directly to obtain $\rho$ (the probability of a spike's being produced), $t_{f}$ (the mean ISI), and $\sigma_{\text {out }}$ (the width of the ISI distribution) as described in section 2.2 . The effect of using this more general synaptic response function, which incorporates a finite rise time of the EPSP, is illustrated in Figure 4, where the output rate of spikes generated is plotted as a function 

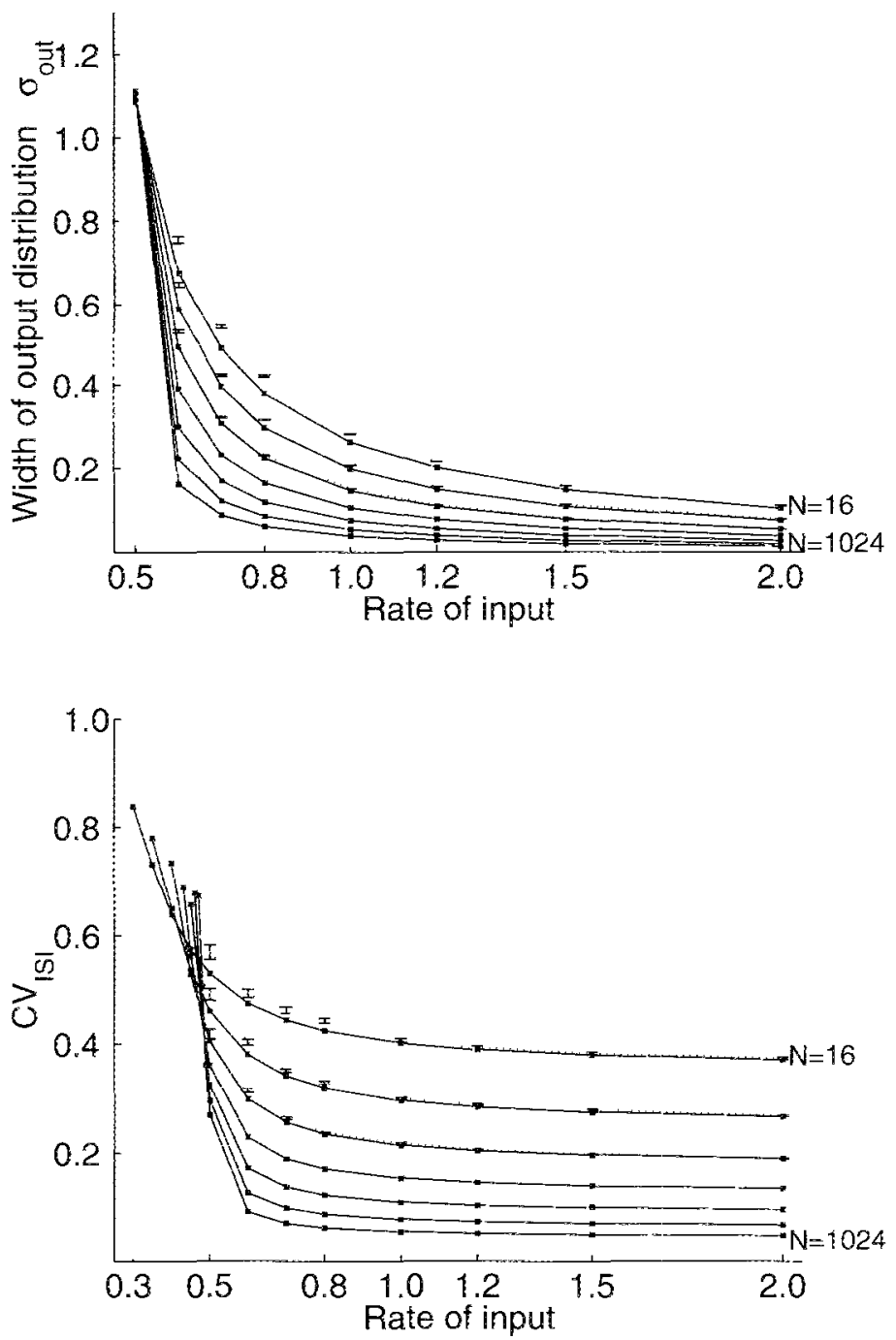

Figure 5: Analytical result for the Stein model using equatıons 2.11 and 3.9 with threshold ratio $R=0.5$ and number of inputs $N=16,32,64,128,256,512,1024$ (lines plotted in order from top to bottom), plotted as a function of the input rate, $\lambda_{1 n}$. (a) Output jitter, $\sigma_{\text {out }}$. (b) Coefficient of variation, equation 3.15. The error bars joined by dotted lines represent the results of numerical simulations involving 10,000 trials. 
of the incoming EPSP's (only excitatory inputs are included here). The solid lines show the results of the Stein model at three threshold ratios, and the dashed lines are the results for the above model with $\alpha=5$ at the threshold ratios $R=0.2$ and 0.5 . The results indicate that the alpha model produces spikes at a lower rate than the Stein model for the same threshold ratio and that the input-output relationship differs substantially from the linear behavior evident for the Stein model. The width of the output spike distribution, $\sigma_{\text {out }}$, was also found to increase relative to that for the Stein model at the same threshold ratio. The value of $\alpha=5$ chosen here is somewhat on the low side of physiologically realistic values, but serves to illustrate the effect of including a finite rise time of the EPSP.

3.4 Distribution of PSP Amplitudes. The expressions for the mean and wid th of the probability density function, equation 2.10, are written in a form that makes their dependence on the distribution of PSP amplitudes transparent, since the functions $D_{k}(t)$ and $E_{k}(t)$ are independent of the amplitude, $a_{k}$. In the discussion so $f_{a r}$, we have considered only that case where the amplitudes are positive (i.e., excitatory PSPs) and equal, $a_{k}=a$. The amplitude of the PSPs, however, will in general depend on their location on the dendritic tree, quantal fluctuations, and other variables (Softky \& Koch, 1993). However, it is straightforward using equation 2.10 to consider the effect of various distributions of amplitudes. In particular, if we consider a distribution of amplitudes, $P(a)$, with mean $a$ and standard deviation $\sigma_{t r}$

$$
\bar{a}=\int_{0}^{x} a P(a) d a, \quad \sigma_{a}^{2}=\int_{0}^{\infty}(a-\bar{a})^{2} P(a) d a,
$$

then the expressions for $\Upsilon(t)$ and $\Gamma(t)$, equation 2.23, which determine the probability density of the potential, become (Stein, 1967)

$$
\begin{aligned}
& \Upsilon(t)=N \bar{a} \lambda \int_{0}^{t} d t^{\prime} u\left(t^{\prime}\right) \\
& \Gamma(t)=N\left(\bar{a}^{2}+\sigma_{a}^{2}\right) \lambda \int_{0}^{t} d t^{\prime} u^{2}\left(t^{\prime}\right) .
\end{aligned}
$$

3.5 Including Inhibition. IPSPS may be included as a special case of the previous section in which the inhibitory terms are negative, $a_{k}<0$. In the simplest situation, there are $N_{E}$ afferent fibers contributing EPSPs of amplitude $a_{E}$ and $N_{I}$ afferent fibers contributing IISSPs of amplitude $a_{I}$ :

$$
\begin{aligned}
& a_{k}=a_{I}>0 \text { for } k=1 \ldots N_{E} \\
& a_{k}=a_{I}<0 \text { for } k=N_{L}+1, \ldots,\left(N_{K}+N_{I}\right)
\end{aligned}
$$


The functions $\Upsilon(t)$ and $\Gamma(t)$, equation 2.10 , then take the form

$$
\begin{aligned}
& \Upsilon(t)=N_{E} a_{E} D_{E}(t) \cdots N_{I} a_{I} D_{l}(t) \\
& \Gamma(t)=N_{E} a_{L}^{2}\left(E_{E}(t)-D_{I}^{2}(t)\right)+N_{i} a_{I}^{2}\left(E_{l}(t)-D_{l}^{2}(t)\right),
\end{aligned}
$$

where $D_{E . I}$ and $E_{E, j}$ are given by equation 2.8 for the excitatory and inhibitory inputs respectively, which may have differing synaptic response functions $u_{E}(t)$ and $u_{l}(t)$.

The effect of including inhibitory inputs is illustrated in Figure 6 for the Stein model, where the lines join the results for particular numbers of inhibitory inputs. The first plot shows the relationship between the input and output rates at a threshold ratio of $R=0.5$ for the case of $N_{t}=64$ excitatory inputs and $N_{I}=0,16,32$, and 48 inhibitory inputs, both of the same magnitude, $a_{E}=a_{1}$. The threshold ratio is defined, as before, just in terms of the excitatory inputs, $R=\theta / N_{F} a_{E}$ (equation 2.2). The plot indicates that the infibitory inputs iower the rate of outputs. The second plot show's the coefficient of variation, equation 3.15 , as a function of the input rate. The error bars joined by the dotted lines for the $N_{I}=0$ and 16 cases represent the results and associated statistical errors of numerical simulations of Poissondistributed inputs involving 10,000 trials. It is observed that introducing inhibitory inputs increases the coefficient of variation of the output spikes, and that this effect is most pronounced near the critical threshold ratio the leftmost points of the plots in. Figure 6).

It has been postulated (Shadlen \& Newrome, 1994) that the inclusion of inhibition is crucial in explaining the apparent discrepancy between the observed irregularity of ISIs in the cortex and the regularity that is evident in models of integrate-and-fire neurons (Softky and Koch, 1992, 1993). The experimentally observed values of the coefficient of variation take values in the range 1.0-1.5 (Shadlen \& Newsome, 1998), as discussed in section 3.2 (although substantially lower values in the range $0.1-0.5$ have been reported when cye movement has been controlled for; (Gur et al., 1997). The integrateand-fire model with only excitatory inputs gives a train of output spikes that are very regular, as illustrated in Figure 5 by the small values of the coefficient of variation in the region substantially above the critical threshold. However, in the parameter region near the critical threshold, the coefficient of variation for the integrate-and-fire model takes values much closer to one, as discussed in section 3.2 and plotted in Figure 5 . The results for including inhibitory inputs are plotted in Figure 6 , which causes an increase in the coefficient of variation, and this increase is iargest near the critical threshold. The results plotted here show values of the coefficient of variation and trends that are in agreement with those in the study of a conductance-based integrate-and-fire model (Troyer \& Miller, 1997): a decrease in the coefficient of variation as the input rate increases and an increase in the coefficient of variation with more inhibitory inputs (the parameters used in their study 
were $\lambda_{i} / \lambda_{E}=0.75, N_{E} \lambda_{E} \approx 140$ spikes $/ \tau, N_{1} \lambda_{3} \approx 65$ spikes $/ \tau$ and their model includes a reversal potential at the reset voltage, which is not included in this analysis).

Of particular interest is the model in which the excitation and inhibition are balanced, which gives rise to values of $C V$ close to one (Tsodyks \& Sejnow'ski, 1995; van Vreeswijk \& Sompolinsky, 1996; Shadlen \& Newsome, 1998). In this balanced situation, the amplitude of the EPSPs and IPSPs may be much larger, of order $1 / \sqrt{N}$, so that the output spike rate is of the same order of magnitude as the input rates and the fluctuations in the voltage remain finite in the large $N$ limit (Troyer \& Miller, 1997; van Vreeswijk \& Sompolinsky, 1998). To model inhibitory inputs accurately, it is necessary to take into account the effect of the reversal potential, which gives the intracellular potential a lower bound and causes the amplitude of the inputs to depend on the current value of the potential (i.e., the PSP amplitude decreases the nearer the membrane potential is to the reversal level). In the simulation of Shadlen and New'some (1998; their case 3, as discussed here in section 3.2 ), the effect of the inhibitory reversal potential is approximated by introducing a lower barrier below which the potential is not permitted to fall. A more complete investigation of the effect of inhibition, which addresses these questions, is beyond the scope of this article, which deals only with synaptic inputs that are summed linearly. Such questions are addressed elsewhere (Burkitt \& Clark, 1999b), where it is shown how the technique presented here for analyzing integrate-and-fire neurons can be extended to include the effect of reversal potentials

\section{Conclusions}

We have presented a new technique for analyzing the behavior of integrateand-fire neurons, which allows both the probability density of the summed potential and the density of cutput spikes (i.e., the ISI distribution) to be calculated. The technique for calculating the probability density, which involves integrating over the arrival times of the input distribution, provides an alternative to the conventional diffusion approximation methods involving random walks or stochastic differential equations, and this new method allows the analysis of both arbitrary input distributions and synaptic response functions (i.e., including rise time and decay of the membrane potential). The output distribution of spikes is given by the first-passage time to threshold, which is defined by an integral relationship involving the first passage time and a conditional probability density. This relationship is a generalization of the renewal equation that holds for both leaky neurons and situations in which there is no time-translational invariance. The technique has been formulated in terms of an arbitrary probability distribution of inputs, and results are derived for inputs that have a Poisson distribution. 

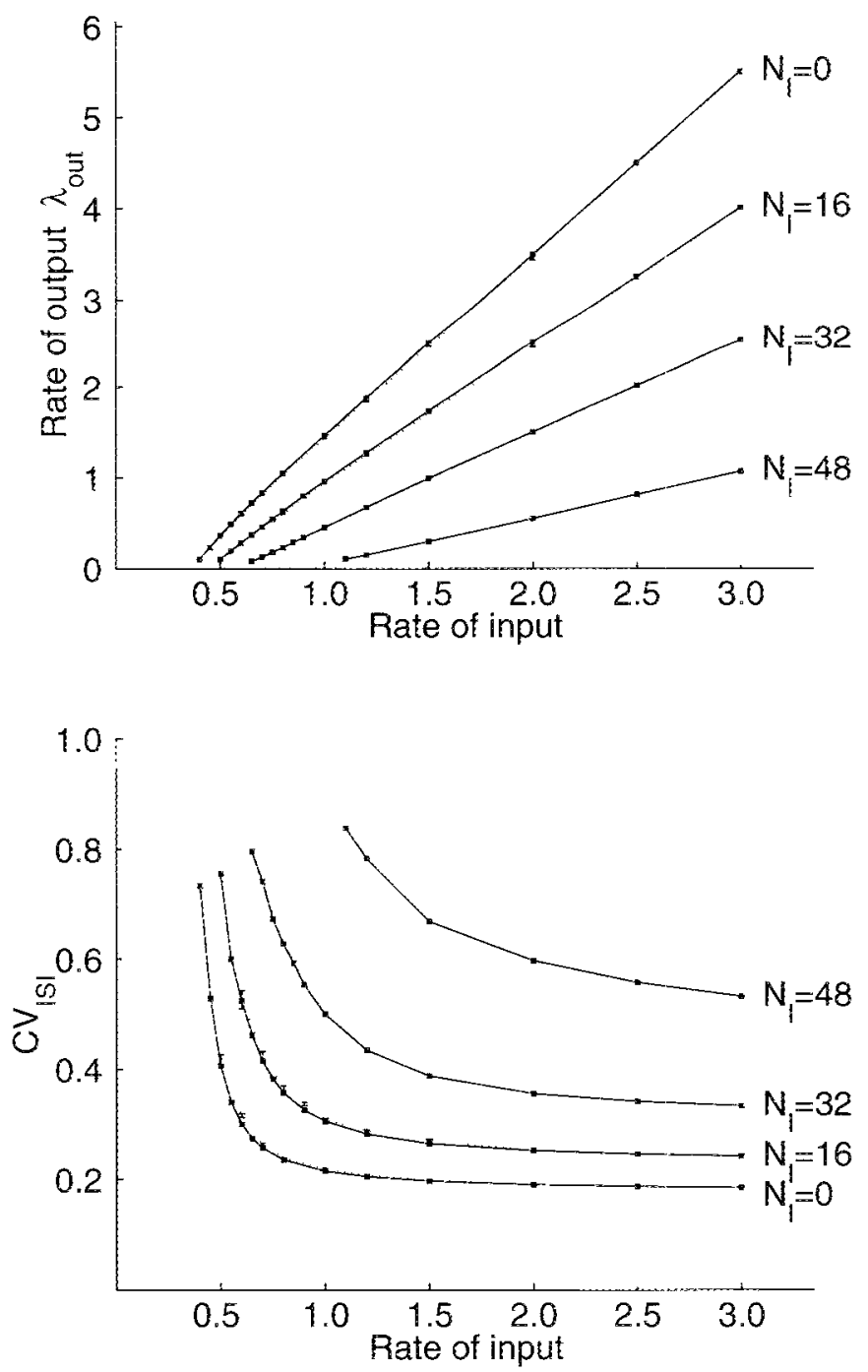

Iigure 6: Results for the Stein model with $N_{F}=64$ excitatory inputs, threshold ratio $R=0.5$, and number of inhibitory inputs $N_{I}=0,16,32$, and 48 , plotted as a function of the input rate, $\lambda_{\mathrm{m}}$. The amplitudes of the EPSPs and IPSPS are equal. (a) Mean output rate, $\lambda_{\text {out }}$. (b) Coefficient of variation of the output spikes, equation 3.15. The error bars joined by dotted lines represent the resuits of numerical simulations involving 10,000 trials. 
The technique is a formal calculation that gives results that coincide with those using the diffusion method for the two cases studied here: the perfect integrator and the Stein model. For the perfect integrator model, the method reproduces the well-known result (Gerstein \& Mandelbrot, 1964). In the case of the Stein model (Stein, 1965), which incorporates decay of the potential, the method reproduces the known solution for the moments of the probability density of output spikes, which are expressed in terms of Laplace transforms of the probability density of the potential and the conditional probability density (Gluss, 1967). Synaptic response functions with finite rise time have also been analyzed, and the moments of the spike distribution, which give, respectively, the probability of an output spike, the average time of spike generation, and the output jitter, have been obtained numerically.

The results are expected to be most accurate when the amplitude of the individual synaptic inputs, $a$, is small in comparison to the threshold, $\theta$ (which is the the difference between the firing and reset potentials), and the number of inputs, $N$, is large (we assume that the amplitude, $a$, scales with $1 / N)$, that is, where a large number of small-amplitude inputs are required to summate in order for the membrane potential to reach the firing threshold. as is the case in many neural systems.

The linear summation of the individual contributions from each synapse in equation 2.1 makes it possible to analyze situations in which a single neuron has a variety of synaptic response functions. Such freedom could be used to model the effect of synapses at different parts of the synaptic tree, whereby synapses nearer the soma would have a synaptic response function with larger amplitude and shorter rise time than synapses farther away. The technique also enables a distribution of PSP amplitudes to be analyzed, including amplitudes that fluctuate randomly about a mean value, which could be used to model the effect of quantal fluctuations.

The relationship between the rate of incoming EPSPs, $\lambda_{1 n}$, and the resultant rate of firing, $\lambda_{\text {out }}$, and the width of the ISl, $\sigma_{\text {out }}$, and the coefficient of variation, $C V$ equation 3.15 , have been analyzed here for the Stein model and the alpha model (which includes both rise time and decay of the membrane potential) for a range of both thresholds and numbers of inputs. The results indicate that the probability of firing, $\rho$, falls from one to zero as the threshold ratio, $R$, increases, and that this decline in the firing probability is sharper as the number of inputs, $N$, increases. This rapid transition from a firing to a nonfiring domain can be characterized by a value of the threshold ratio, $R_{\text {crit }}$, which depends principally on the input rate. In the cases investigated here, the smali value of the coefficient of variation, $\mathrm{CV}$, above the critical threshold indicates that the output distribution is not Poissonian in this range of parameters, but rather is much more regular than typically observed in cerebral cortex (Softky \& Koch, 1992, 1993). However, the results here indicate that near the critical threshold, the coefficient of variation, $\mathrm{CV}$, has values much closer to those experimentally observed. An alternative 
explanation involves the effect of inhibitory inputs (Shadlen \& Newsome, 1994), and studies using this technique do indeed show that the coefficient of variation increases when IPSPS are included. A more comprehensive examination of thus issue incorporating reversal potentials (Tuckweli, 1979; Hanson and Tuckwell, 1983; Musila \& Lánsky, 1994) for the inhibitory PSPs is under way to examine this possibility (Burkitt and Clark, 1999b).

\section{Appendix A: Evaluation of $D_{k}(t)$ and $E_{k}(t)$}

The functions $D_{k}$ and $E_{k}$, equation 2.8 , may be evaluated using Laplace transforms, which are indicated by the subscript $L, p_{k, L}(s)=\mathcal{L}\left\{p_{k}(t)\right\}$, as follows,

$$
D_{k L}(s)=u_{k, L}(s) \sum_{m=1}^{\infty} p_{k_{, L}}^{m_{L}}(s)=\frac{p_{k, I}(s)}{1 \cdots p_{k L}(s)} u_{k . L}(s)
$$

Where the convolution property of Laplace transforms is repeatedly applied.

The expression for $E_{k}$ is separated into a diagonal part $\left(m=m^{\prime}\right)$ and an off-diagonal part $\left(m \neq m^{\prime}\right)$ that are evaluated independently. The diagonal term is evaluated as above for $D_{k, L}(s)$ (except now with $u_{h}^{2}(t)$ ). The lower and upper off-diagonal terms are equal and consist of two infinite sums, each of which may be successively treated in the same way as above:

$$
\begin{aligned}
E_{k, L}(s)= & \mathcal{L}\left\{u_{k}^{2}(t)\right\} \sum_{m=1}^{x} p_{k . L}^{m}(s) \\
& +2 \sum_{m-1}^{\alpha} p_{k . I}^{m}(s) \mathcal{L}\left\{u_{k}(t) \mathcal{L}^{-1}\left\{u_{k . L}(s) \sum_{m i=1}^{\alpha} p_{k, I}^{m^{\prime}}(s)\right\}\right\} \\
= & \frac{p_{k L}(s)}{1-p_{k . L}(s)} \mathcal{L}\left\{u_{k}^{2}(t)\right\}+2 \frac{p_{k, I}(s)}{1-p_{k, I}(s)}-\mathcal{L}\left\{u_{k}(t) D_{k}(t)\right\}
\end{aligned}
$$

For a Poisson process, these expressions take particularly simple forms, as shown in section 2.3 .

\section{Appendix B: Evaluation of $G\left(t_{2} . t_{1}\right)$}

As in the case of the above expression for $L_{k L}$, equation A.2, we separate $G\left(t_{2} . t_{1}\right)$ into diagonal $\left(n=m^{\prime}\right)$ and off-diagonal $\left(n \neq m^{\prime}\right)$ parts that are evaluated independently. The diagonal term is evaluated in a similar fashion to the above, except now the Laplace transforms are carried out with respect to $t_{1}$, and the diagonal term is $u\left(t_{1}\right) u\left(t_{1}+\Delta\right)$, where $\Delta=t_{2}-t_{1} \geq 0$ is treated as a parameter. The lower and upper off-diagonal terms are equal and consist of two infinite sums, each of which may be successively treated 
in the same way as in appendix $A$ :

$$
\begin{aligned}
G_{L}(s: \Delta)= & \mathcal{L}\left\{u\left(t_{1}\right) u\left(t_{1}-\Delta\right)\right\} \sum_{m_{L}=1}^{x} p_{L}^{m}(s) \\
& +2 \sum_{m=1}^{x} p_{L}^{m_{2}}(s) \mathcal{L}\left\{u\left(t_{1}\right) \mathcal{L}^{-1}\left\{u_{I}\left(s^{\prime}\right) \sum_{m^{\prime}=1}^{\infty} p_{L}^{m^{\prime}}\left(s^{\prime}\right)\right\}\right\} \\
= & \frac{p_{L}(s)}{1-p_{L}(s)} \mathcal{L}\left\{u\left(t_{1}\right) u\left(t_{1}-\Delta\right)\right\} \\
& -2 \frac{p_{L}(s)}{1 \cdots p_{L}(s)} \mathcal{L}\left\{u\left(t_{1}\right) L\left(t_{1}+\Delta\right)\right\} .
\end{aligned}
$$

where $s$ and $s^{\prime}$ refer to the Laplace transform with respect to $t_{1}$ and $t_{2}$, respectively. For a Poisson process, these expressions take particularly simple forms (see section 2.3).

\section{Acknowledgments}

This work was funded by the Cooperative Research Centre for Cochlear Implant, Speech and Hearing Research. We thank the annnymous referees for their useful comments on the manuscript.

\section{References}

Abeles, M. (1982a). Local cortical circuits: An electrophysiologral study. Berlin: Springer-Verlag.

Abeles, M. (1982b). Role of the cortucal neuron: Integrator or coincidence detector? Isr. J. Meid. So , 18, 83-92.

Abeles, M. (1991). Corticonics: Neural arcuts of the cerebrat cortex. New York: Cambridge Lniversity Press.

Adrian, F. (1928). The basis of sensation: The action of sense urgans. London: Christophers

Bernander, O., Koch, C., \& Usher, M. (1994). The effect of synchronized inputs at the singie neuron level. Neural Comput., 6, 622-641.

Bialek, W., \& Rieke, I. (1992). Reliability and information transmission in spiking netirons. Trends Neitrosci., 15, 428-434.

Burkitt, A. N., \& Clark, G. M. (1999a). Analysis of integrate-and-fire neurons: Synchronization of synaptic input and spike output in neural systems. Neural Comput., 11, 871-901.

Burkitt, A. N., \& Clark, G. M. (1999b). Balanced networks: Analysis of ieaky integrate and fire neurons with reversal potentials. Submitted.

(arr. C. F., \& Friedman, M. (1999). Fvolution of time coding systems. Neural Comput, 11, 1-20.

Cope, D. K., \& Tuckwell, H. C. (1979). Firing rates of neurons with random excitation and inhibition. J Theor. Biol., 80, 1-14. 
Diesmann, M., Gewaltig, M. O., \& Aertsen, A. (1996). Characterization of synfire activity by propagating "pulse packets". In J. Bower (Ed.), Computational ncuroscience: Trends in research (pp. 59-64), San Diego: Academic Press.

bouglas, R., \& Martin, K. (1991). Opening the grey box. Trends Neurosci, 14, $286-293$.

Engel, A. K., König, P., Kreiter, A. K., Schillen, T. B., \& Singer, W. (1992). Temporal coding in the visual cortex: New vistas on integration in the nervous system. Trends Neurosit. 15, 218-226.

Gabbiani, F., \& Koch, C. (1996). Coding of time-varying signals in spike trains of integrate-and-fire neurons with random threshold. Neural Comput., 8, 44-66.

Gerstein, G. 1.., \& Mandelbrot, B. (1964). Random walk models for the spike activity of a single neuron. Biophys. I., 4, 41-68.

Gerstner, W. (1995) Time structure of the activity in neural network models. Phys. Rev. F, 51, 738-758.

Gluss, B. (1967). A model for neuron firng with exponential decay of potential resulting in diffusion equations for probability density. Bull. Math. Broplysics, $29,233-243$.

Gur, M., Beylin, A., \& Snodderly, D. M. (1997). Response variability of neurons in primary visual cortex (V1) of alert monkeys ]. Neurosit., 17, 2914-2920.

Hanson, F. B., \& Tuckwell, H. C. (1983). Diffusion approximations for neurona? activity including synaptic reversal potentials. J. Theoret. Neurobiol., 2, 127153

Hopfield, J. J. (1995). Pattern recognition computation using action potential timing for stimulus representation. Nature, 376, 33-36.

Iyengar, S., \& Liao. Q. (1997). Modeling neural activity using the generalized inverse gaussian distribution. Biol. Cybem., 77, 289-295.

Jack, J. J. B., Noble, D., \& Tsien, R. W. (1985). Electric cument flow in excitable cells. Oxford: Clarendon Press.

Judd, K. T., \& A Ahara, K. (1993) Pulse propagation networks: A neural network model that uses temporal coding by action potentials Neural Networks, 6 , 203-215.

Kistler, W. M., Gerstner, W., \& van Hemmen, J. L. (1997). Reduction of the Hodgkin-Huxley equations to a single-variabie threshold model. Neural Comput., 9, 10i5-1045.

Koch, C. (1999). Biophysics of computation. Infornation processing mingle netirons. Oxford: Oxford University Press.

Lanský, P. (1984). On approximations of Stein's neuronal model. J. Theor. Biol., $107.631-647$

Lapicque, L.. (1907). Recherches quantitatives sur l'excitation electrique des nerfs traitée comme une polarization. ]. Physiol. (Paris), 9. 620-635.

Maass, W. (1996a). Lower bounds for the computational power of netwrorks of spiking neurons. Neural Comput., 8, 1-40.

Maass, W. (1996b). Networks of spiking neurons. In P. Bartlett, A. Burkitt, \& R. C. Williamson (Fds.), Proceedings of the Seventh Austrainan Conference on Neural Networks. Canberra: Australian National University.

Maršálek, P., Koch, C., \& Maunsell, J. (1997). On the relationship between synaptic input and spike output jitter in individual neurons. Proc. Natl. Acad Sa. 
USA, 94, 735-740.

Musila, M., \& Lánský, P. (1994). On the interspike intervals calculated from diffusion approximations of Stein's neuronal model with reversal potentials. J. Theor. Biol, 171, 225-232.

Paolmi. A. G., Clark, G. M., \& Burkitt, A. N. (1997). Intracellular responses of rat cochlear nucleus to sound and its role in temporal coding. NeuroReport, $8(15), 3415-3422$.

Papoulis, A. (1991). Probabulity, random oariables, and stochastic processes (3rd ed.), Singapore: McGraw-Hill International Editions.

Plesser, J. E., \& Tanaka, S. (1997). Stochastic resonance in a model neuron with reset. Phys. Lett A, 225, 228-234

Press, W. H , Flannery, B P., Teukolsky, S. A., \& Vettering, W. T. (1992). Numerwal rectpes th Fortran: The art of scientific computmg. Cambridge: Cambridge University Press.

Rhode, W.S., \& Smith, P.H. (1986). Fncoding tiring and intensity in the ventral cochlear nucleus of the cat. 1. Neurophystol, 56, 261-286.

Shadlen, M. N., \& Newsome, W T. (1994). Norse, neurd codes and cortical organization. Curr. Opin. Neurobioi., 4, 569-579.

Shadien, M. N., \& Newsome, W. T. (1998). The variable discharge of cortical neurons Implications for connectivity, computation, and information coding. I. Neturosci., 18, 3870-3896.

Singer, $W$. (1993). Synchronization of cortical activity and its putative role in information processing and learning. Annu. Rev. Plysiol, 55, 349-374.

Softky, W. R. (1994). Sub-millisecond coincldence detection in active dendritic trees. Neurosat. 58, 13-41.

Softky, W. R., \& Koch, C. (i992). Cortical cells should fire regularly, but do not. Neural Comput , 4, 643-646

Softky, W. R., \& Koch, C. (1993). The highly irregular firing of cortical cells is inconsistent with temporal integration of random FPSPs. ]. Neurosct, 13, $334-350$

Stein, R. B. (1965). A theoretical analysis of neuronal variability. Btophy, I., 5, $173-194$.

Stein, R. B. (1967). Some models of neuronal variability. Btophys. J., 7, 37-68.

Theunissen, F., \& Miller, J. P. (1995). Temporal encoding in nervous systems: A rigorous definition. 1. Comput. Ne'trosct., 2, 149-162.

'Troyer, T. W. \& Miller, K. D. (1997). Physiological gain leads to high IsI variability in a simple model of a cortical regular spiking cell Neural Comput., 9, 971-983

Tsodyks, M. V., \& Sejnowski. T. I. (1995). Rapid state switching in balanced cortical network models Network, 6, 111-124.

Tuckwell, 1. C. (1977). On stochastic models of the activty of single neurons. I. Theor. Biol. 65,783-785.

Tuckwell, H. C. (1978). Neuronal interspike time histograms for a random input model. Brophys ]., 21, 289-290.

Tuckwell, 31. C. (1979). Synaptic transmission in a model for stochastic neural activity 7 . Theor. Btol., 77. 65-81.

Tuckwell, II C. (1988a). Introduction to theoretical neurobnology: Vol. 1, Lmear cable theory and dendritu structure. Cambridge: Cambridge University Press. 
Tuckwell, H. C. (1988b). Introduction to theoretical neurobiology: Vol 2, Nonlinear and stochastic theories Cambridge: Cambridge University l'ress.

Tuckwell, H. C. (1989). Stochastic processes in the netirusctences. Philadelphia: Society for Industrial and Applied Mathematics.

Tuckweil, H. C., \& Cope, D. K. (1980). Accuracy of neuronal interspike times calculated from a diffusion approximation. J. Theor. Btol, 83, 377-387.

van Vreeswijk, C., \& Sompolinsky, H. (1996). Chaos in neuronal networks with balanced excitatory and inhibitory activity. Sclence, 274, 1724-1726.

van Vreeswijk, C., \& Sompolinsky, H. (1998). Chaotic balanced state in a model of cortical circuits. Neural Comput., 10, 1321-1371.

Wilbur, W. J., \& Rinzel, J. (1982). An analysis of Stein's model for stochastic neuronal excitation. Biol. Cybern., 45, 107-114.

Recesved April 28, 1948, accepted June 7, 1999. 


\section{University Library}

\section{- M M I N E R VA A gateway to Melbourne's research publications}

Minerva Access is the Institutional Repository of The University of Melbourne

\section{Author/s:}

Burkitt, A. N.;Clark, Graeme M.

Title:

Calculation of interspike intervals for integrate-and-fire neurons with Poisson distribution of synaptic inputs

Date:

2000

\section{Citation:}

Burkitt, A. N., \& Clark, G. M. (2000). Calculation of interspike intervals for integrate-and-fire neurons with Poisson distribution of synaptic inputs. Neural Computation, 12(8), 1789-1820.

Persistent Link:

http://hdl.handle.net/11343/28755 